\section{Correlative Electron Tomography And Elemental Microanalysis In Biology: A Preview}

Michael Marko, Terence Wagenknecht, and Carmen Mannella Resource for Visualization of Biological Complexity, Wadsworth Center marko@wadsworth.org

\section{Introduction}

The combination of electron tomography (three-dimensional reconstruction of an individual object from a series of EM tilt images) and elemental mapping (based on element-specific differences in interaction between the primary electron beam and the specimen) is at the very beginning of its application. This article reviews the first published results and considers some requirements and potential uses in biology, employing two examples from our own exploratory work.

Electron tomography has been in use in the biological sciences for over 30 years, although it has become widespread only in the last several years. A review of electron tomography from the biological perspective appeared in the July/August 2003 issue of this magazine. The reader is referred there for background and additional references, and also to a standard reference (1) and an excellent summary introduction (2). The use of electron tomography in materials science is just beginning, but has already led to approaches for obtaining 3-D compositional as well as structural information. These approaches are also applicable to biology. An important factor in the increasing use and range of applications of tomography has been the commercial availability of improved EM hardware and control software. This has led to turnkey systems for fully automated tomographic data-collection, and makes it feasible to integrate electron tomography and elemental microanalysis.

There are three main approaches for combining tomography and microanalysis:

(i) A complete tomographic tilt series of elemental maps can be recorded. If contrast between different elements is required to see the structure of interest, tomographic reconstruction from elemental maps is the only way to fully reveal the $3-\mathrm{D}$ shape. For example, the work of the Bazett-Jones laboratory $(3 ; 4)$ shows that domains within the cell nucleus, not distinguishable using conventional TEM with general heavy-metal staining, can be visualized by comparing phosphorus maps (indicating presence of nucleic acid) and nitrogen maps (representing protein). In principle, this work could be extended to 3-D using tomography.

(ii) A 2-D elemental map can be compared to a conventional (i.e., brightfield TEM) tomogram. If the 3-D shape of objects in the specimen can be determined by the tomogram, a 2-D elemental map will often be sufficient for identification of elemental composition of the objects. A stereo-pair of elemental maps may help in some cases (in fact, in the case of a specimen consisting of a sparse array of discrete particles, a stereo-pair of elemental maps might suffice for determination of both the 3-D distribution and the elemental composition of the particles).

(iii) If an object in a conventional tomogram is well-defined and homogeneous, its elemental content can be determined from a single microanalytic spectrum recorded from a selected area of the object.

The microanalytic methods so far applied to electron tomography are electron energy-loss spectroscopy (EELS), energy-dispersive $x$ ray spectroscopy (EDX), and high-angle annular darkfield scanning transmission EM (HAADF-STEM). EELS (except for low-loss EELS), and EDX are based on the interaction of the primary electron beam with an atom's core electrons (5). The primary electrons undergo an element-specific loss of energy in the specimen. In EELS, the energies of the transmitted beam are dispersed by a magnetic prism to pruviue a spectrum, displayed as energy loss relative to the primary beam energy. The different elements appear as edges in the EELS spectrum. If an energy-selecting slit is placed after the prism, electrons from a specific element can be mapped as an energy-filtered TEM (EFTEM) image. Some of the energy lost in the specimen takes the form of $x$-rays with element-specific energies. The $x$-rays are detected in the vicinity of the specimen, and an EDX spectrum shows peaks representing $X$-rays detected at various energies. In HAADF-STEM, primary electrons that are scattered at high angles by passing close to the atomic nucleus are detected (2). The scattering angle increases with atomic number (Z). The camera length of the EM is adjusted so that the HAADF STEM detector captures electrons scattered within a defined angular range, and an image from the high-Z element is formed. Using STEM mode, EELS and EDX spectra can be collected at each pixel of an image. This technique is known as spectrum imaging (SI). An advantage of SI is that maps of individual elements can be extracted off-line once the data is collected. Since a spectrum image is already three-dimensional ( $x$, $y$, and energy), a tomographic reconstruction from a tit series of such images would be four-dimensional.

The first tomographic reconstructions from tilt series of elemental maps were reported at the Microscopy and Microanalysis 2001 meeting by materials science groups in the U.K. (6-8). The laboratory of Möbus and Inkson, at the time at Oxford, pioneered EFTEM and STEM-mode EDX tomography (9), and the laboratory of Midgley and Weyland at Cambridge pioneered HAADF-STEM tomography (2). The first biological application of HAADF-STEM tomography was by Koster's group at Utrecht (10), and the first biological application of EFTEM tomography was at Leapman's laboratory at NIH (11).

A basic requirement for tomographic reconstruction is that the contrast in the projection (tilt-series) images represents a monotonically varying function through the specimen (12). At moderate resolution in biology, this is almost always the case. Exceptions arise in the case of crystalline specimens with a small unit cell, in which case diffraction contrast is very strong, and is dependent on the tilt of the specimen relative to the crystal planes. Another exception, most noticeable at fairly high magnification using a highly coherent illumination system such as field-emission-gun, is Fresnel contrast, which changes with depth in the specimen and with specimen tilt. Diffraction and Fresnel contrast can be avoided by incoherent imaging, which can be achieved, for example, by using HAADF-STEM or EFTEM (2), or hollow-cone illumination (9). The specimen thickness for EELS should be limited to not more than one inelastic mean-free path. The mean-free path of plastic is about $100 \mathrm{~nm}$ at $100 \mathrm{kV}$ and $250 \mathrm{~nm}$ at $400 \mathrm{kV}$. For ice, the values are about 1.5 times higher. Aside from these considerations, the choice of microanalytic mode is based on the radiation sensitivity of the specimen, the element(s) of interest, the amount of the element present, and the spatial resolution required. The specimen preparation method also plays an important part.

\section{Modes}

EFTEM is probably the easiest mode to use for biological work involving mapping of lighter elements, and is the mode most readily applicable to approach (i), above, i.e. tomographic reconstruction from elemental maps. Zero-loss EFTEM imaging has been used for several years to improve contrast in electron tomography of frozen-hydrated specimens (13), so there is a solid base of relevant experience. The whole image is collected at once, and data collection is generally faster than with the STEM-based modes. Spatial resolution is high, and the image size can be large. For EFTEM tomography, standard automated tilt-series collection software (see below) needs only to be modified for 


\section{RESEARCH TECHNICIAN/ ELECTRON MICROSCOPY}

Howard Hughes Medical Institute, a leading biomedical research organization, is seeking a Research Technician with experience in electron microscopy for our site at the Yale University School of Medicine.

The laboratory focuses on research in cellular neurobiology, with emphasis on synaptic cell biology. Responsibilities include: Conduct experiments independently after discussion of the plan with supervisor, interpret experimental results in consultation with supervisor and collaborators in the lab. Manage an electron microscope and assist in the management of light microscopes, prepare specimens and sections for light and electron microscopy, observe samples, collect images, store and process them either via digital or conventional photographic techniques. Train lab personnel in electron microscopy techniques. The job will offer opportunities for professional growth and the successful candidate could learn and apply cell biological techniques beyond microscopy.

Requirements include a bachelor's degree, knowledge of histology and experience in electron microscopy. Good organizational and analytical skills are essential.

HHMI offers a competitive salary and an excellent benefits package. Please send your resume and cover letter to: $\mathrm{Dr}$. Pietro De Camilli, Howard Hughes Medical Institute, Yale University School of Medicine, 295 Congress Avenue, New Haven, CT 06510 (e-mail: vikki.spelta@yale.edu). EOE.

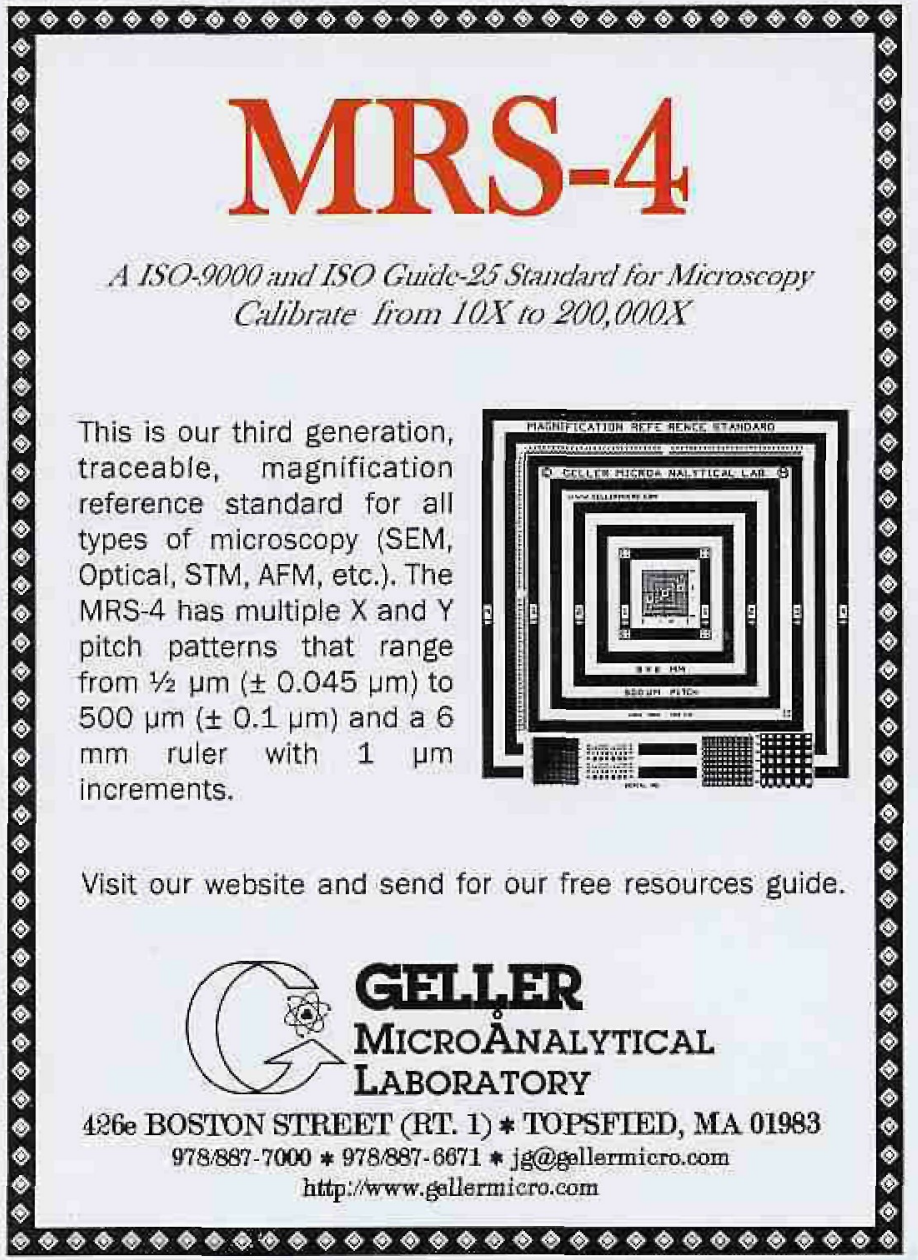

\section{If you have a microscopy application...}
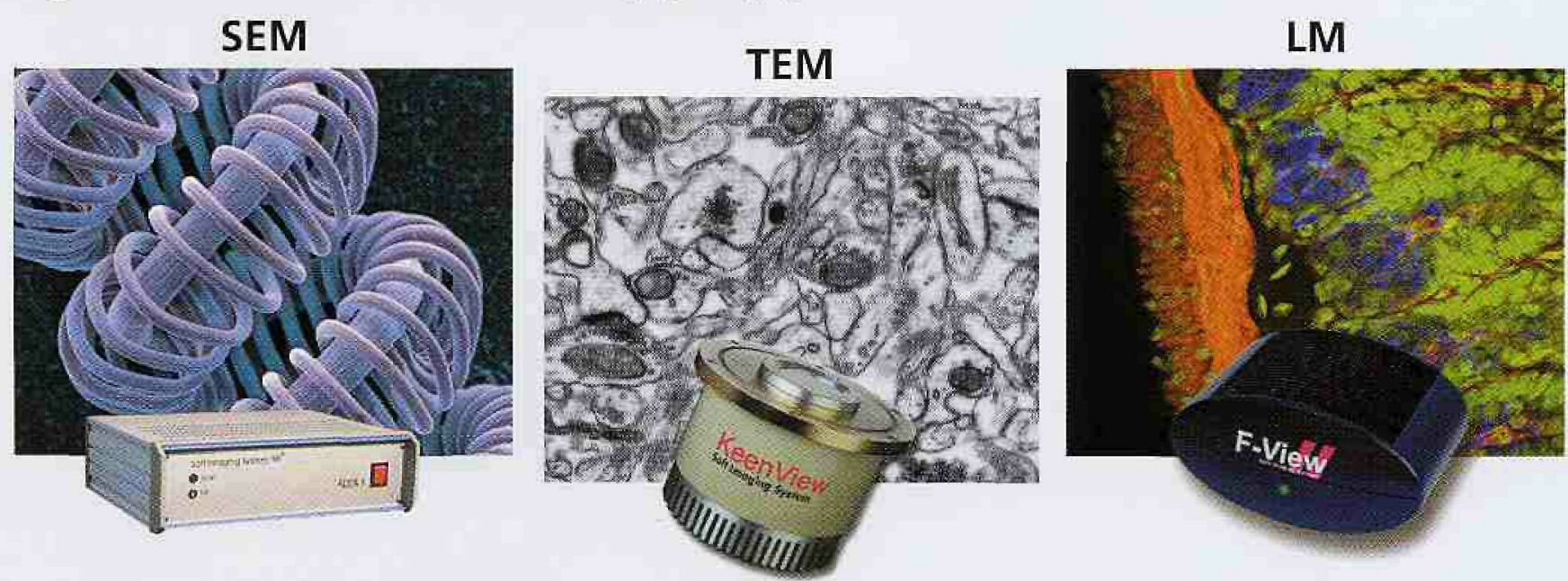

Soft Imaging System offers high resolution, high sensitivity, easy to install cameras for Transmission Electron Microscopes, and Light Microscopes. A digital beam interface is offered for Scanning Electron Microscopes. All of our hardware systems integrate with analysiS ${ }^{\circ}$ software - an image analytical system that provides you with microscope control, camera operation, optimized image acquisition, manual or autornated image analysis, database archiving, report generation and more.

...we have your solution.

Digital Solutions for Imaging and Microscopy Soft Imaging System

\begin{tabular}{|c|c|c|c|}
\hline For defalled information please contast & $\begin{array}{l}\text { Soft Imaging System } \\
\text { inforisoft-imaging.com } \\
\text { Www soft-imagingicom }\end{array}$ & $\begin{array}{l}\text { Narth Amienice } \\
\text { Europe } \\
\text { Asial / Pacific }\end{array}$ & $\begin{array}{l}(888) \text { FIND S15 } \\
(303) 23 n-9270 \\
+49(251) 79800-0 \\
+60(3) 8313-1400\end{array}$ \\
\hline
\end{tabular}




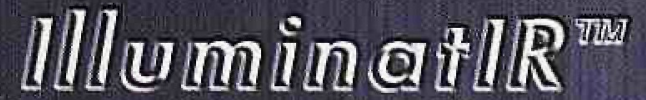
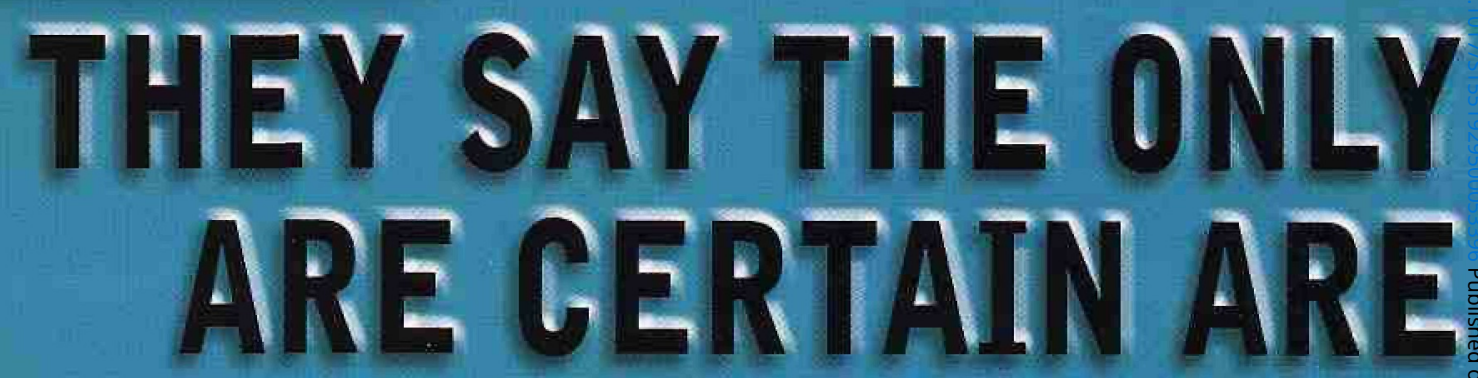

WITH THE ILLUMINATIR,

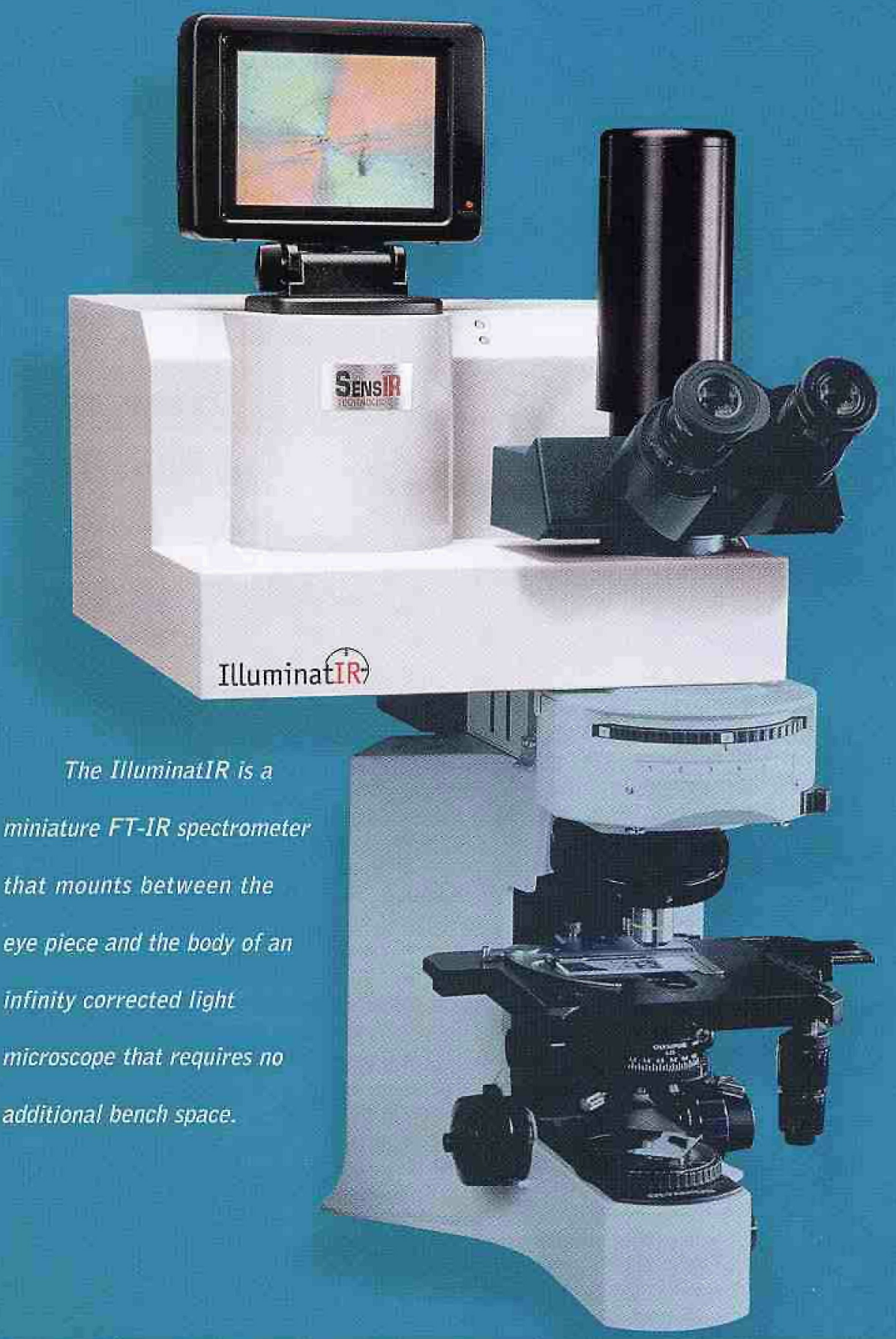




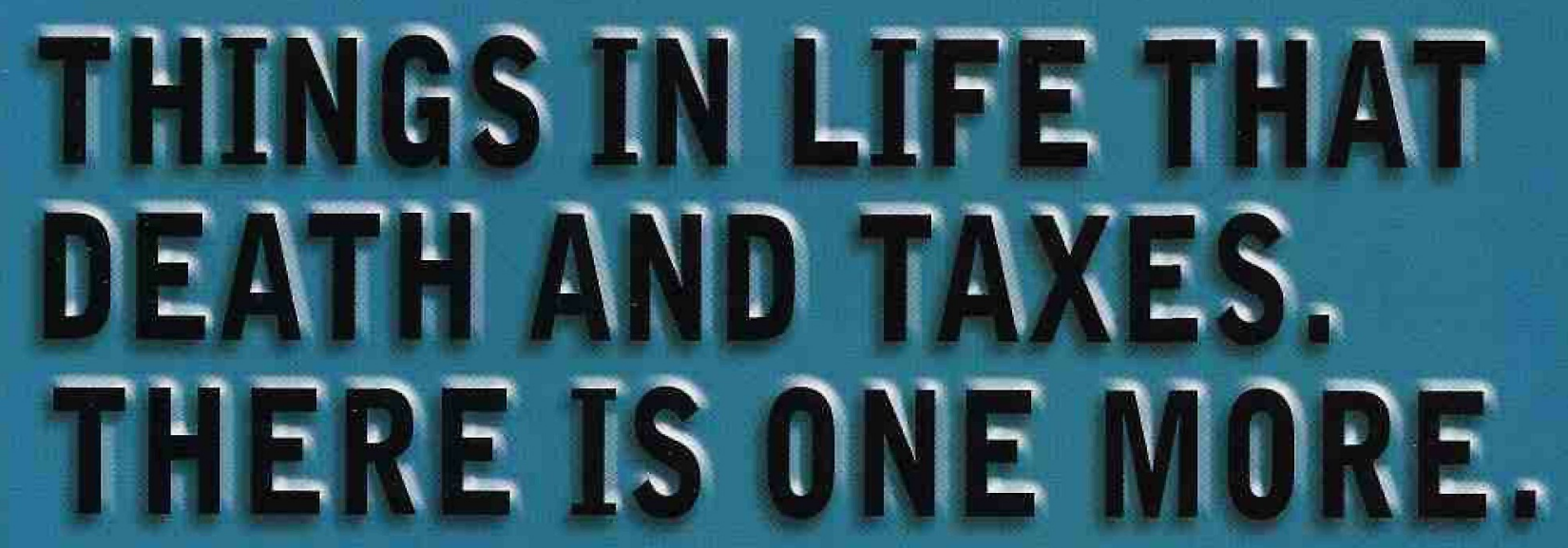

\section{IlluminatIR adds the power of FT-IR analysis to your light microscope}

Introducing the IlluminatIR, the only miniaturized FT-IR system that attaches directly to your favorite light micnoscope. Add the power of molecular analysis to your visual interpretation for the most accurate and objective results.
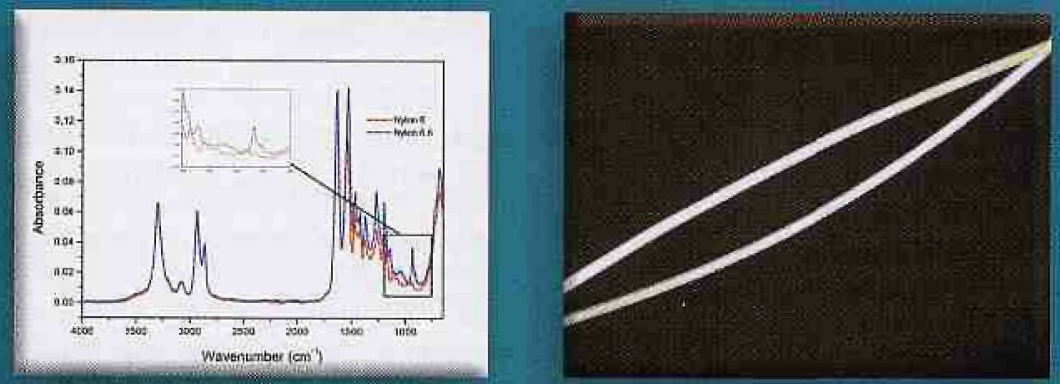

When samples are Visually identical, FT IR microscopy can show you the difference. Visually thise two nylon fibers are identical. However when FTt/R analysis is run on each of the fibers and their spedtra is overlald the differente in the twa flacers becomes alear. The top fiber was identified in Nylan 6 and the bottem fiber to be Nylon 6,6 .

Only IlluminatIR:

- provides high quality infrared data and superior visual quality

- easily attaches to infinity corrected microscopes

- maintains all the capabilities of your microscope, i.e. polarization, fluorescence, Nomarski or image analysis, etc.

- can run FT-IR analysis simply with the turn of an objective - no need to move the sample

- adds these capabilities without taking up additional bench space

- very easy to use and maintain

The IlluminatIR: it will change the way microscopes are used forever.

\section{MAKE YOUR OWH OBJECTIVE OPINION.}

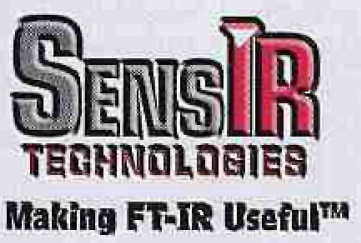




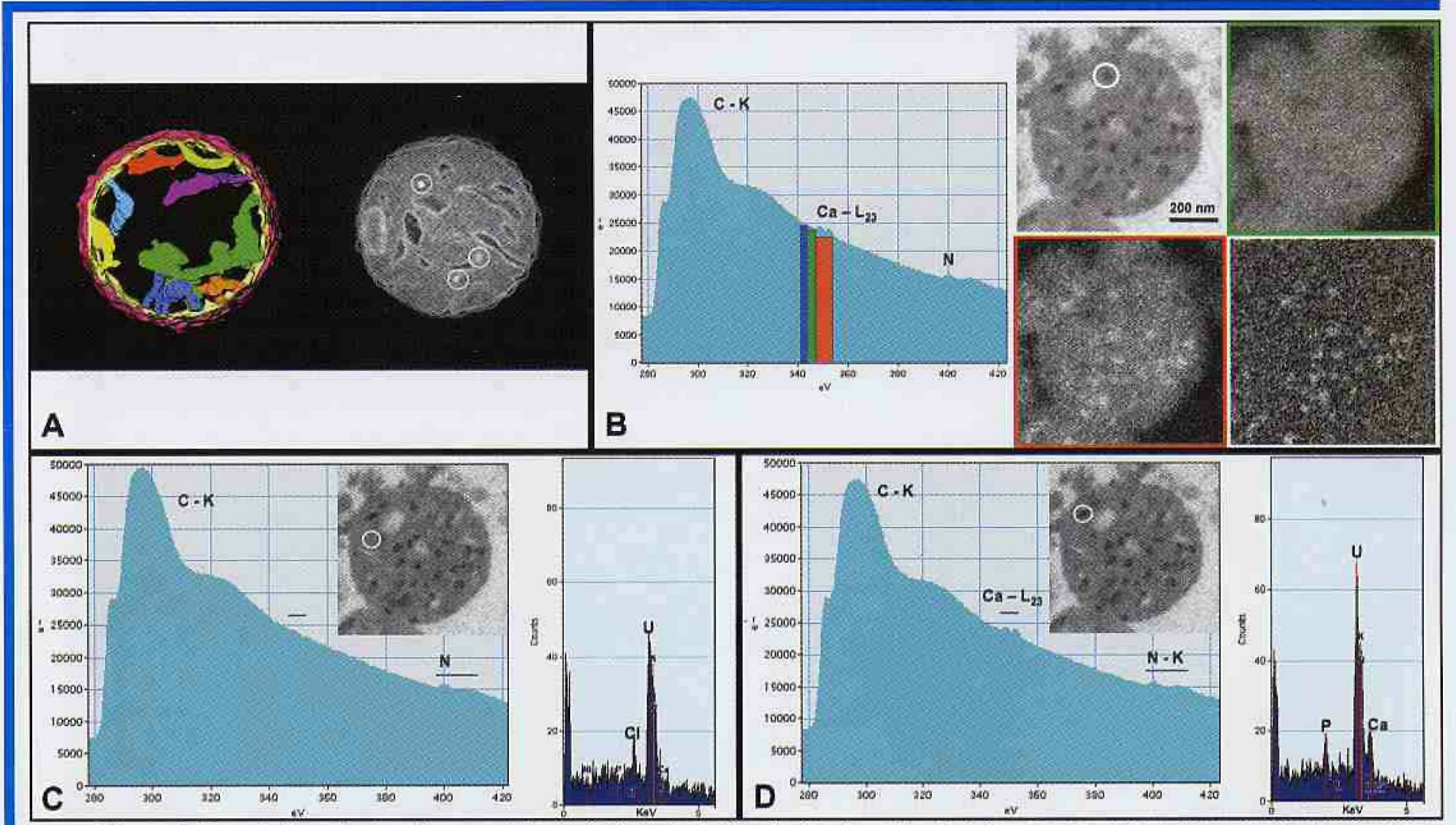

Figure 1. Correlative electron tomography and microanalysis of mitochondria: Calcium content of matrix granules in plastic sections of isolated rat-liver mitochondria (29). (A) Tomogram of conventionaliy-fixed isolated mitochondrion; surface rendering of inner and outer membranes and selected cristae (left); matrix granules (circled) in thin slice through tomogram (right). (B) EFTEM calcium map of mitochondrion prepared with anhydrous embedding (14), including Os fixation and $U$ and Pb post-staining. EELS spectrum from $50 \times 50 \mathrm{~nm}$ area containing single matrix granule (circled at right in zero-loss image). Calcium map (lower right) created by background subtraction based on two pre-edge images (blue and green bars on spectrum, and green-outlined image) and a post-edge image (red bar and red-outlined image). Exposure time 40 s for each image. Strong calcium edge in raw spectrum proves validity of EFTEM mapping. Tomography would be feasible. (C) EELS (left) and X-ray (right) spectra collected simultaneously from circled area of mitochondrial matrix lacking granules. No visible Ca edge under bar in EELS spectrum; Cl peak but no visible Ca or P peaks in EDX spectrum. U peak from post-stain. (D) EELS spectrum from circled granule shows $C, C a$, and $N$ edges; EDX shows $C a, P$, and $U$ peaks. EELS $P$ edge, offscale at $132 \mathrm{eV}$, would not be visible, as explained in text. Ca concentration in granule $100 \mathrm{mmol} / \mathrm{Kg}$ dry weight, from EELS according to method in (16). JEOL JEM4000FX at 400kV, with Gatan GIF 2002, Noran Be-window EDX detector, Emispec ES Vision microanalysis system. Section thickness $100 \mathrm{~nm}$.

recording a series of images with various energy offsets at each tilt. A low-loss image is useful for focusing and tracking after each tilt. For each element to be mapped, background subtraction requires one or two pre-edge images, and one post-edge image. Two pre-edge images are required for quantitative elemental analysis; this is known as the three-window method (11; Fig. 1B). However, the jump-ratio method, which requires only one pre-edge image, can offer better contrast ( 3 ; 9). In order to ensure that an EFTEM map is trustworthy, an EELS spectrum should be acquired from an appropriately selected area of the specimen, to ensure that the desired edge is present.

EELS (usually performed in STEM mode) gives the highest sensitivity for most trace elements of biological relevance. When using EELS spectrum imaging, a complete EELS spectrum is collected at each pixel in the image. This enables use of better methods for background correction and extraction of weak signals than are available in EFTEM. Acquisition time per image is often on the order of minutes, and increases with image size, so a typical spectrum image is rarely larger than $512 \times 512$ pixels. While the long acquisition time (and correspondingly high electron dose) would make tomographic reconstruction impractical for some specimens, correlation between a single or stereo-pair EELS spectrum image and a tomogram (approach ii, above) should be feasible in many cases. When EELS is used in the selected-area mode (approach iii), an EELS spectrum is recorded from

a defined area of the specimen, and this is correlated with a particular object in a conventional tomogram. The size and position of the area to be analyzed can be easily selected and monitored during spectrum acquisition by simultaneous observation of the annular darkfield STEM image. We have found the EELS selected-area mode to be very useful for determining, at high sensitivity, the elemental composition of an object within a specimen (Fig. 1C,D and Fig. 2).

EDX spectroscopy is inherently less efficient than EELS. In EELS, most of the energy-loss electrons are forward scattered and reach the EELS spectrometer, while in EDX the $x$-rays are emitted in all directions, and many do not enter the detector. The acquisition time per image using EDX SI is likely to be very long. In the first proof of concept for EDX tomography (a materials science application using a very radiation-resistant specimen), only 11 elemental maps were recorded as tilt images, with an image size of $256 \times 256$ pixels, and an acquisition time of 20 min per map (9). Nevertheless, with some specimens, (see the example of phosphorus, below), EDX offers an advantage, and will at least be useful for approaches (ii) and (iii). Note that simultaneous spectrum imaging with EELS and EDX provides complementary information (Fig. 1C,D).

HAADF-STEM is ideal for mapping elements with high atomic number. For tomography, the tilt-series acquisition time is reasonable since only a STEM image--not a spectrum image--needs to be collected 


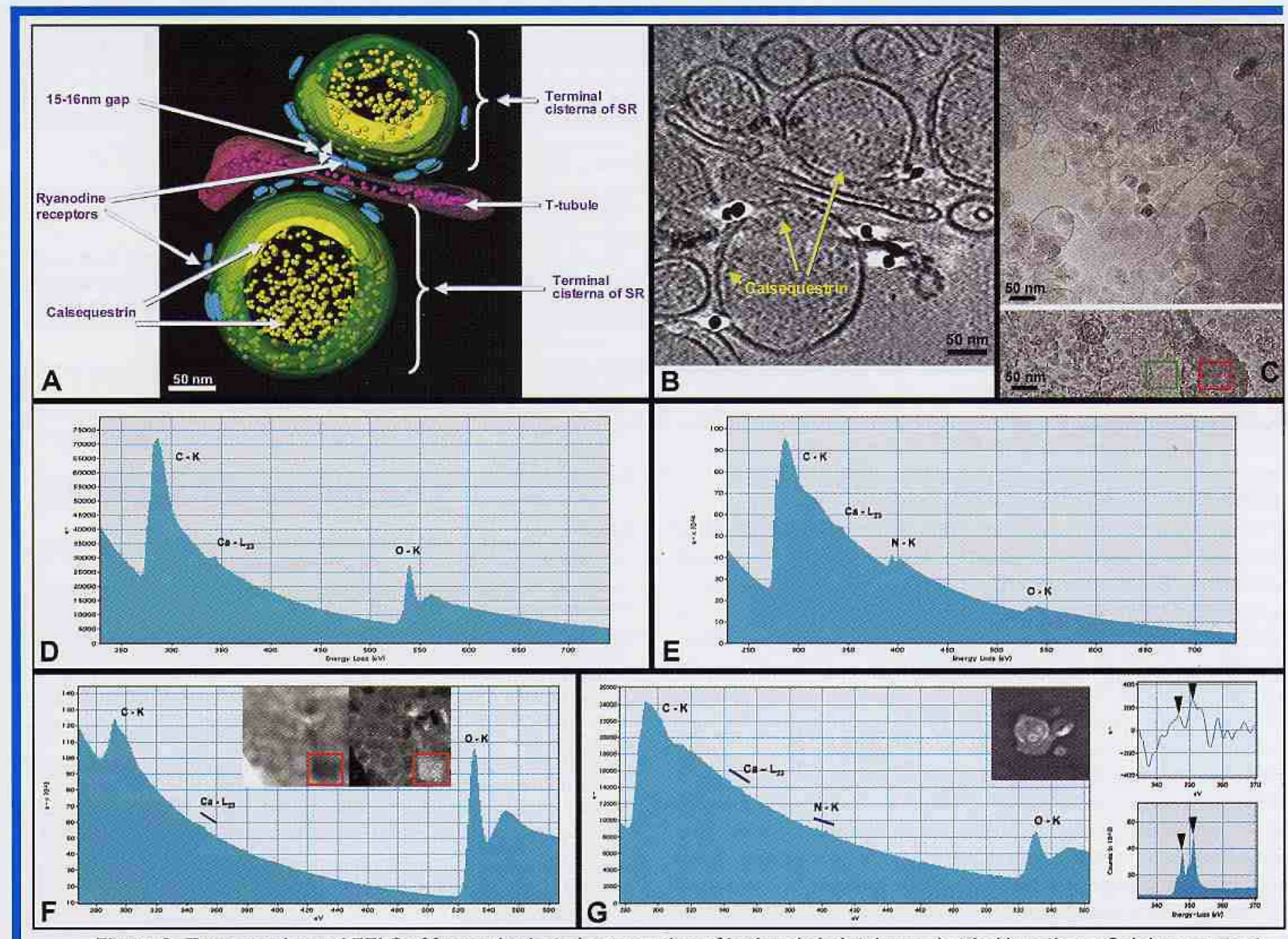

Figure 2. Tomography and EELS of frozen-hydrated suspension of isolated skeletal muscle triad junctions: Calcium content in sarcoplasmic reticulum (SR) vesicles. (A) Surface-rendered model from electron tomography showing ryanodine receptors (RyR, calcium-release channels) adjacent to material believed to be the protein calsequestrin inside SR vesicle; granular material inside SR vesicle may also contain $\mathrm{Ca}^{++}(30)$. (B) Thin slice from tomogram in (A). (C) Zero-loss phase-contrast images of Ca+-loaded frozenhydrated SR and vesicles before (top); and after (bottom) freeze-drying. (D) EELS spectrum from green box in (C). Note overall lower intensity than (E). C and $O$ edges are from Formvar support film. Ca from precipitated buffer corresponds to concentration of 10 mmol/Kg dry wt. (E) EELS spectrum at SR vesicle, red box in (C). C and $\mathrm{N}$ signals are mainly from vesicle. Ca content is $150 \mathrm{mmol} / \mathrm{Kg}$ diry wt. EFTEM tomography should be possible. (F) Core-loss EELS analysis of frozen-hydrated specimen. Inset shows darkfield STEM (left) and zero-loss EFTEM (right) images of field of buffer precipitate particles. Analyzed area $50 x 50$ nm, red-boxed at lower right. Large 0 edge proves presence of ice. Ca concentration $\sim 10 \mathrm{mmol} / \mathrm{kg}$ dry wt., electron dose $10^{6} \mathrm{e} / \mathrm{hm}^{2}$. Correlative selected-area microanalysis or STEM-EELS spectrum imaging after frozen-hydrated tomography should be feasible. (G) EELS analysis of freeze-dried SR vesicle, shown in inset by darkfield STEM. Ca concentration at normal physiological level, estimated to be $\sim 1 \mathrm{mmol} / \mathrm{Kg}$ dry wt. Ca edge not visible in raw spectrum; but can be extracted from the background $(16 ; 31)$. Extracted Ca edge (upper right) matches example from EELS atlas. (lower right). EFTEM mapping not feasible, but EELS spectrum imaging possible (13). JEOL JEM4000FX at $400 \mathrm{kV}$, with Gatan GIF 2002, Gatan EELS analysis software. Original ice thickness of all specimens is $150 \mathrm{~nm}(\sim 0.4$ inelastic mean-free path).

at each tilt. The spatial resolution can be high and the image can be large. In its first biological application, tomographic reconstruction from a tilt-series of HAADF-STEM images was shown to be useful for visualizing nanogold immuno probes in plastic sections (10). At high resolution, dynamic focusing is needed to maintain focus across an image of a highly-tilted specimen (2); this feature can be added to the tilt-series acquisition software.

\section{Specimens}

Sections of plastic-embedded biological specimens have the advantage of being able to withstand the very high electron dose often needed for microanalysis. EFTEM with plastic sections is ideal for mapping relatively plentiful elements in biological specimens such as nitrogen and phosphorus $(3,4)$. The first EFTEM tomographic recon- struction was a 3-D phosphorus map of the cytoplasm of a nematode cell, showing the RNA-containing ribosomes (11). For EELS/EFTEM of phosphorus, it is important to omit osmium and uranium from the preparation (3). Osmium contributes to a diffuse background in the region of the phosphorus $\mathrm{L}_{2,3}$ edge (132 eV), and the tail of the uranium $\mathrm{O}_{4,5}$ edge at $96 \mathrm{eV}$ also increases the background for phosphorus. High-pressure freezing can be used to improve structural preservation in the absence of osmium (11). Avoiding osmium and uranium is not required for EELS/EFTEM of calcium ( $350 \mathrm{eV}$ ) and nitrogen (400 eV), as seen in Fig 1. Osmium and uranium do not interfere with phosphorus mapping using EDX (Fig $1 \mathrm{C}, \mathrm{D}$ ). Diffusible jons such as $\mathrm{Na}^{+}, \mathrm{K}^{+}$, and $\mathrm{Ca}^{++}$can be studied in plastic sections by using anhydrous processing, although ultrastructural preservation is not ideal (14; Fig. 1). Better preservation can be obtained by plastic embedding after freeze-drying 
and osmium vapor treatment (15). If the elemental signal is too weak to make a complete tilt series practical, good correlation between a conventional tomogram and a stereo-pair elemental map using EELS or EDX spectrum imaging should be possible, because (after brief preirradiation) there is negligible collapse of the specimen during either tomography or microanalysis. For even greater sensitivity or accuracy in quantification, correlation can be made with selected areas using STEM /EELS or EDX.

Frozen-hydrated (quick-frozen) biological specimens offer obvious advantages for both structural studies and elemental microanalysis: they lack chemical fixatives or stains, retain diffusible elements in place, and avoid dehydration and resin embedment. Use of frozen-hydrated tissue sections has become a standard TEM method for biological microanalysis $(16 ; 17)$. Freeze-drying in the EM before analysis is usually required due to the very high electron dose needed for an elemental map (but see Fig. 2F). Electron tomography of frozen-hydrated specimens was pioneered by the Baumeister lab at Martinsried, Germany (18); here in Albany, we pioneered electron tomography of frozen-hydrated tissue sections (19). We are investigating how microanalysis can be combined with tomography of frozen-hydrated specimens. EELS water mapping can be done on frozen-hydrated specimens $(20 ; 21)$ since water can be mapped using the low-loss signals from outer-shell electrons. The high signal levels in this region of the EELS spectrum allow use of a low electron dose, and at least a limited tilt serles of low-loss elemental maps should be possible. For core-loss elemental mapping, the electron dose needed for even a single 2-D elemental map $\left(10^{7}-10^{9} \mathrm{e} / \mathrm{nm}^{2}\right)$ far exceeds the limit of detectible structural damage for a frozen-hydrated specimen (about $10^{5} \mathrm{e} / \mathrm{nm}^{2}$ ). Although freeze-dried tissue sections or suspensions retain recognizable structure, there is considerable collapse, and some congealing, of the cellular contents. An obvious strategy would be to record a tomogram while the specimen is frozen-hydrated, then freeze-dry in the EM and record a 2-D elemental map for correlation. In some cases, however, we are finding that it is difflcult to re-locate or, in the case of small features, even to recognize the same object after drying. We have found that we can do core-loss STEM-EELS analysis on selected areas of frozen-hydrated specimens (Fig. 2F). Although the selected area is damaged, a direct correlation with the tomogram of the frozen-hydrated specimen can be made, and a clear record is left of the area that was analyzed.

\section{Instrumentation and software}

Investigators with access to a modern analytical TEM/STEM instrument can undertake correlative tomography and microanalysis with minimal additional investment. The maximum specimen tilt should be at least $60^{\circ}$, which may not be possible with the existing objective polepiece. For our LEO TEM, we overcame a tilt limitation problem with a special high-tilt holder (22). It is also possible to modify a standard specimen holder in-house (2). Holder modifications are also needed for EDX so that $x$-rays can be collected over a wide specimen tilt range (9). Tilt series can be acquired completely manually, at least for radiation-resistant specimens such as plastic sections, but software for automated tilt-series collection is highly desirable. In our laboratory we use FEl tomography software (23) for our Tecnai TEM, in-house modified Emispec tomography software (24) for our JEOL TEM, and TVIPS software (25) for JEOL and LEO TEMs. For the special applications of tomography discussed here, the user will likely need to develop custom routines for processing and aligning tilt-series images and computing the reconstructions. The two UK materials science groups reported here (2; 9) used IDL (26). We use SPIDER (27). For general tomographic image processing, IMOD (28) is widely used.

\section{References}

The Resource for visualization of Biological Complexity is supported by NIH NCRR Biomedical Research Technology Program Grant RR01219, and the Wadsworth Center EM core. We thank Dr. F. Ichas of the University of Bordeaux, France and Dr. P. Bernardi of the University of Padua, Italy for the anhydrously processed mitochondria samples.

1. Frank J, Ed. (1992) Electron Tomography. Plenum, New York.

2. Midgley PA, Weyland M (2003) 3D electron microscopy in the physical sciences: the development of Z-contrast and EFTEM tomography. Uttramicroscopy $96(3-4): 413-431$

3. Bazett-Jones DP, Hendzel MJ (1999) Electron spectroscopic imaging of chromatin. Methods 17(2):188-200.

4. Boisvert F-M, Hendzel MJ, Bazett-Jones DP (2000) Promyelocytic leukemia (PML) nuclear bodies are protein structures that do not accumulate RNA. $J$ Cell Biol 148:283-292.

5. Egerton RF (1996) Electron energy-loss spectroscopy in the electron microscope (second edition) Plenum, New York

6. Weyland M, Midgley PA, Thomas, JM (2001) High angle annular dark field (HAADF) STEM tomography of nanostructured catalysts. Microse Microanal 7 (Suppl 2 Proceedings): 1104-1105.

7. Weyland M, Midgley PA (2001) Three dimensional energy-filtered transmission electron microscopy (3D-EFTEM). Microsc Microanal 7 (Suppl 2 Proceedings): $1162-1163$.

8. Möbus G, Inkson BJ (2001) Electron spectroscopic tomography for materials science. Microsc Microanal 7 (Suppl 2 Proceedings): $84-85$.

9. Möbus G, Doole RC, Inkson BJ (2003) Spectroscopic electron tomography. Uitramicroscopy 96(3-4):433-451.

10. Zlese U, Kubel C, VerkleijA, Koster AJ (2002) Three-dimensional localization of ultrasmall immuno-gold labels by HAADF-STEM tomography. J Struct Biol $138(1-2): 130-136$.

11. Laquerriere $P$, Kocsis $E$, Zhang $G$, Talbot, TL, Leapman RD (2003) Elemental tornography of biological structures. Microse Microanal 9 (Suppl 2: Proceedings): $240-241$.

12. Hawkes PW (1992) The electron microscope as a structure projector, in: J Frank (Ed.) Electron Tomography Plenum, New York.

13. Grimm R, Koster AJ, Ziese U, Typke D, Baumeister W (1996) Zero-loss energy filtering under low-dose conditions using a post-column energy filter. $J$ Microsc 183:60-68.

14. Manston $J$, Katchburian $E$ (1983) Demonstration of mitochondrial mineral deposits in osteoblasts after anhydrous fixation and processing. $J$ Microsc 134(2): 177-182.

15. Pezzati $R$, Bossi M, Podini P, Meldolesi J, Grohovaz F (1997) High-resolution calcium mapping of the endoplasmic reticulum-golgi-exocytic membrane system: Electron energy loss imaging analysis of quick frozen-freeze dried PC12 cells. Mol Biol Cell 8:1501-1512.

16. Leapman RD, Hunt JA, Buchanan RA, Andrews SB (1993) Measurement of low calclum concentration in cryosectioned cells by parallel-EELS mapping. Uttramicroscopy 49:225-234.

17. Somlyo AP, Bond M, Somlyo AV (1985) Calcium content of mitochondria and endoplasmic reticulum in liver frozen rapidly in vivo. Nature 314:622-625.

18. Dierksen K, Typke D, Hegerl R, Walz J, Sackmann E, Baumeister W (1995) Three-dimensional structure of lipid vesicles embedded in vitreous ice and investigated by automated electron tomography. Blophys $J 68(4): 1416-1422$.

19. Hsieh C-E, Marko M. Frank J, Mannella CA (2002) Electron tomographic analysis of frozen-hydrated tissue sections. J Struct Biol 138:63-73.

20. Sun SQ, Shi S-L, Hunt JA, Leapman RD (1995) Quantitative water mapping of cryosectioned cells by electron energy-loss spectroscopy. J Microsc 177(1):18-30.

21. Aitouchen $A$, Shi S, Libera M, Misra M (2002) Mapping inter-cellular water in skin. Microsc Microanal 8 (Suppl 2 Proceedings):284-285.

22. www. fischione.com/Brochures/tomoholder.htm

23. www.feic.com/tecnai/main,htm

24. www.ernispec.com

25. www.tvips,com

26. www.rsinc.com

27. www.wadsworth.org/spider doc

28. http://bio3d.colorado.edu/imod/

29. Marko M, Wagenknecht TW, Ichas F. Bernard P. Mannella CA (2003) Correlative Electron Tomography and Elemental Microanalysis in Biology. Microsc Microanal 8 (Suppl 2 Proceedings): $1180 \mathrm{CD}$

30. Wagenknecht $T$, Hsieh C-E, Rath BK, Fleischer S, Marko M (2002) Electron tomography of frozen-hydrated isolated triad junction. Biophys. J. 83:24912501.

31. Shuman $H_{1}$ Somlyo AP (1987) Electron energy loss analysis of near-traceelement concentrations of calcium. Ultramicroscopy 21:23-32. 


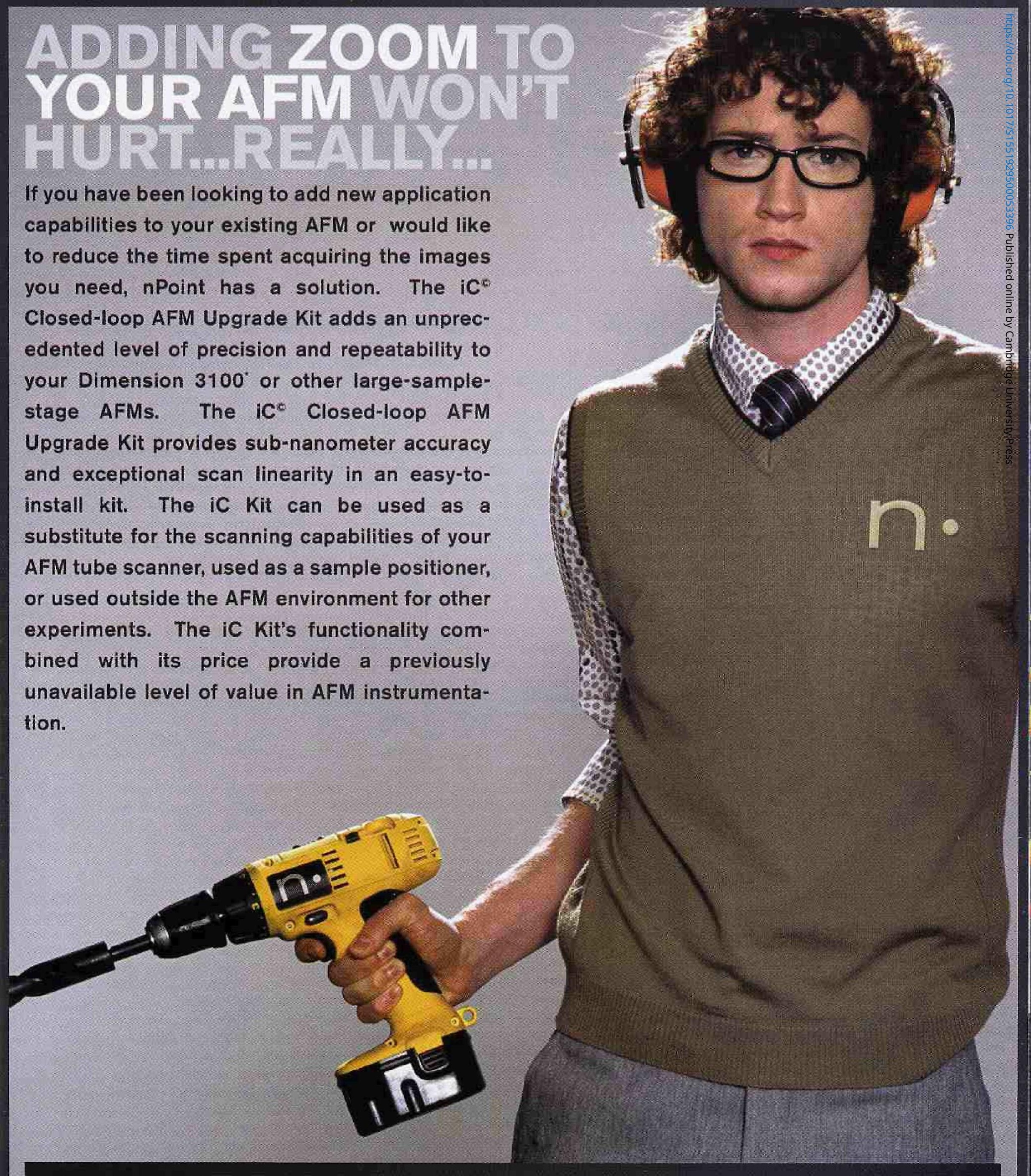

FOR MORE INFORMATION:

www.npoint.com

sales@npoint.com

(608) 204.8758

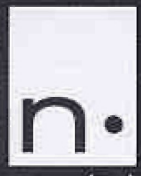

rpoint 